\title{
Expanding functional protein sequence space using generative adversarial networks
}

\author{
Donatas Repecka ${ }^{* 1}$, Vykintas Jauniskis ${ }^{* 1}$, Laurynas Karpus ${ }^{\star 1,2}$, Elzbieta Rembeza ${ }^{3}$, Jan Zrimec ${ }^{3}$, Simona \\ Poviloniene ${ }^{4}$, Irmantas Rokaitis ${ }^{1,4}$, Audrius Laurynenas ${ }^{4}$, Wissam Abuajwa ${ }^{5}$, Otto Savolainen ${ }^{5}$, Rolandas \\ Meskys $^{4}$, Martin K. M. Engqvist ${ }^{3}$, Aleksej Zelezniak ${ }^{3,6,+}$ \\ 1 - Biomatter Designs, Sauletekio al. 7, LT-10257, Vilnius, Lithuania \\ 2 - Institute of Biotechnology, Life Sciences Center, Vilnius University, Saulètekio al. 7, LT-10257, Vilnius, \\ Lithuania \\ 3 - Department of Biology and Biological Engineering, Chalmers University of Technology, Kemivägen 10, \\ SE-412 96, Gothenburg, Sweden \\ 4 - Institute of Biochemistry, Life Sciences Center, Vilnius University, Saulètekio al. 7, LT-10257, Vilnius, \\ Lithuania \\ 5 - Chalmers Mass Spectrometry Infrastructure, Chalmers University of Technology, Kemivägen 10, \\ SE-412 96, Gothenburg, Sweden \\ 6 - Science for Life Laboratory, Tomtebodavägen 23a, SE-171 65, Stockholm, Sweden \\ *Equal contribution \\ ${ }^{+}$corresponding author (email: aleksej.zelezniak@chalmers.se)
}

\begin{abstract}
De novo protein design for catalysis of any desired chemical reaction is a long standing goal in protein engineering, due to the broad spectrum of technological, scientific and medical applications. Currently, mapping protein sequence to protein function is, however, neither computationionally nor experimentally tangible ${ }^{1,2}$. Here we developed ProteinGAN, a specialised variant of the generative adversarial network ${ }^{3}$ that is able to 'learn' natural protein sequence diversity and enables the generation of functional protein sequences. ProteinGAN learns the evolutionary relationships of protein sequences directly from the complex multidimensional amino acid sequence space and creates new, highly diverse sequence variants with natural-like physical properties. Using malate dehydrogenase as a template enzyme, we show that $24 \%$ of the ProteinGAN-generated and experimentally tested sequences are soluble and display wild-type level catalytic activity in the tested conditions in vitro, even in highly mutated (>100 mutations) sequences. ProteinGAN therefore demonstrates the potential of artificial intelligence to rapidly generate highly diverse novel functional proteins within the allowed biological constraints of the sequence space.
\end{abstract}




\section{MANUSCRIPT}

A protein's three-dimensional structure, physicochemical properties and molecular function are defined by its amino acid sequence. From the 20 commonly occurring proteinogenic amino acids, a small sized protein comprising 100 amino acids can be made in $10^{130}$ unique ways. In this vast multidimensional space - often referred to as the protein fitness landscape ${ }^{4}$ - as little as 1 in $10^{77}$ sequences are estimated to fold into the defined three-dimensional structures to carry out specific functions ${ }^{5-7}$. This imposes a great burden on experimental approaches aiming to design novel protein sequences, such as random mutagenesis ${ }^{4}$ and recombination of naturally occurring homologous proteins ${ }^{8,9}$, as up to $70 \%$ of random amino acid substitutions typically result in a decline of protein activity and $50 \%$ are deleterious to protein function ${ }^{4,10-16}$. On the other hand, Artificial intelligence ( $\mathrm{Al}$ ) is not limited by the amount of sequence variations it can process ${ }^{17-19}$ and, instead of depending on a blind search, is based on an inference-based process - it infers protein properties ${ }^{18,20}$ and function ${ }^{19,21}$ directly from training examples. Recent Al approaches have also demonstrated great potential in capturing both the structural and evolutionary information found in natural protein sequences ${ }^{17,22}$. Nevertheless, the majority of existing machine learning models in biology are discriminative ${ }^{17,18,21,23}$, i.e. the model is trained using readily available data, to predict the properties of a given protein sequence. A generative modeling approach, in contrast, could generate new sequence samples from the learned portion of functional protein sequence space, providing direct access to unexplored sequence diversity within functional protein structures, without the need to test a large number of non-functional protein sequence variants. Indeed, breakthrough generative learning strategies, such as Generative Adversarial Networks (GANs) ${ }^{3}$ can learn multidimensional distributions in disparate scientific domains to generate photorealistic images ${ }^{24}$, hand-written text ${ }^{25}$, music ${ }^{26}$ and even DNA sequences with biological properties ${ }^{27,28}$. Hence, in the present study we develop and test ProteinGAN (Figure 1a) to learn and sample the protein sequence space for de novo generation of functional enzymes.

ProteinGAN is a generative model architecturally tailored specifically to learn patterns in long biological sequences (Methods, Supplementary Figure 1). A customized temporal convolutional network ${ }^{29}$ enabled the network to simultaneously analyze local and global sequence fragments, and thus to capture the meaningful sequence motifs and long-distance relationships that are critical for correct protein structural assemble. Additionally, we introduced a self-attention layer ${ }^{30}$ to help ProteinGAN focus on functionally important areas across the entire length of the sequences, such as catalytic residues (Methods, Supplementary Figure 1). The final architecture of the network comprised 45 layers with over 60 million trainable parameters.

A family of bacterial malate dehydrogenase $(\mathrm{MDH})$ enzymes (EC 1.1.1.37) was used to train the neural network. MDH is a tricarboxylic acid cycle enzyme catalyzing the conversion of malate to oxaloacetate using $\mathrm{NAD}^{+}$as a cofactor (Supplementary Figure 2). We chose MDH based on the following criteria: (i) it 
has a large number of diverse sequences (a total of 16706 unique sequences were used for training), which were on average $319 \pm 18.2$ (sd) amino acids long with pairwise sequence identities as low as $10 \%$; (ii) it is a complex enzyme that must bind both its substrate and the NAD ${ }^{+}$cofactor for catalysis and (iii) its activity can be readily monitored in vitro. During model training, we assessed the quality of the sequences generated by ProteinGAN at every 1200 learning steps. In each assessment, 64 sequences were generated and their identities to natural sequences in the training and validation datasets were computed (Figure 1b). After 2.5M learning steps, at which training was terminated (Supplementary Figures 3,4$)$, the mean sequence identities between the generated and natural sequence sets had reached a plateau (median sequence identity to the closest natural sequences was $61.3 \%$ ). Following the initial quality assessment, 20000 of the generated sequences were used to further evaluate the ProteinGAN performance.

a

Random
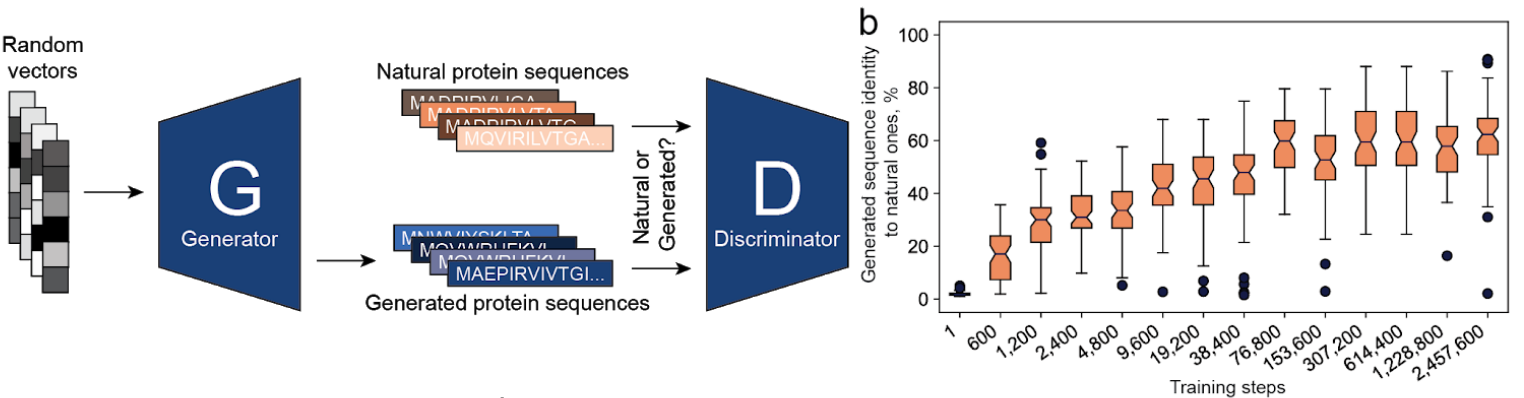

C
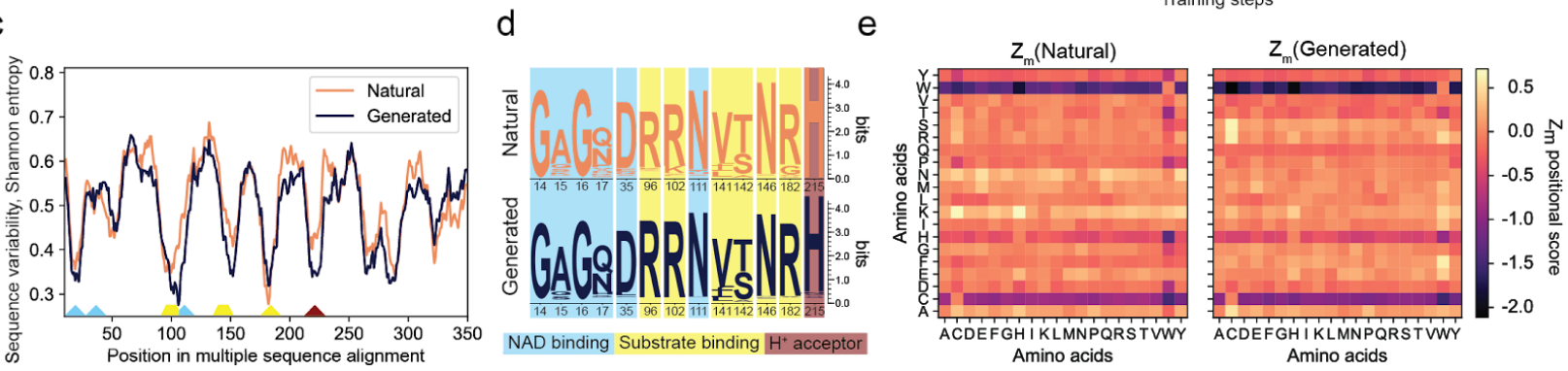

Figure 1. | ProteinGAN learns intrinsic relationships of natural protein sequences. a) ProteinGAN training scheme. Given a random input vector, the Generator network produces a protein sequence which is scored by the Discriminator network comparing it to the natural protein sequences. The generator tries to fool the discriminator by generating sequences that will eventually look like real ones (the generator never actually sees real enzyme sequences). b) Sequence identity of 64 generated sequences to the nearest natural sequence at different training timestamps. c) ProteinGAN effectively captures amino acid distribution of natural MDH sequences. Sequence variability expressed as Shannon entropies for generated and training sequences estimated from multiple-sequence alignment (MSA). Low Shannon entropy values represent highly conserved and thus functionally relevant positions, whereas high entropy indicates high amino acid diversity at a given position. d) A sequence logo of key conserved positions in the multiple sequence alignment. e) ProteinGAN learns the order of amino acids in natural MDH sequences. Amino acid pair association $\left(Z_{m}\right.$ positional score) matrices for Natural and Generated protein sequences. Positive values indicate a larger distance than expected when comparing random sequences with the same amino acid frequency. The numbers indicate how many positions, on average, the amino acids in a pair are closer (negative values) or further apart (positive values) than in a random sequence. 
First, we evaluated ProteinGAN's ability to capture evolutionary sequence properties, i.e. the positional amino acid variation in generated and natural sequences. Shannon entropies were computed for each position in multiple sequence alignments of the generated and natural MDH sequences (Figure 1c). The positional variability in generated sequences was highly similar to that in natural sequences, with peaks (high entropy) and valleys (low entropy) appearing at similar positions in the sequence alignment. Indeed, we observed a high correlation (Pearson's $r=0.89, p$-value $<1 \mathrm{e}-16$ ) between the entropy values of generated and natural sequences. At conserved positions the generated sequences preserved key substrate-binding and catalytic residues (Figure 1d). Further comparative analysis of generated and natural sequences showed that even in highly variable sequence regions, the frequencies of individual amino acids were perfectly correlated (Pearson's $r=0.96, p$-value $<1 \mathrm{e}-16$, Supplementary Figure 5). Moreover, for each individual sequence, ProteinGAN inferred the specific physicochemical amino acid signatures present in this enzyme class. For instance, despite high sequence diversity among generated sequences, the fractions of hydrophobic, aromatic, charged and cysteine-containing residues were the same (Wilcoxon rank sum test $p$-value $>0.05$ ) as in natural ones. Apart from the differences in hydrophilic and polar uncharged residues ( $p$-value $=7 e-5$ and $1 \mathrm{e}-28$, respectively), the network had learned the overall amino acid composition corresponding to both the evolutionary and physicochemical constraints (Figure 1c, d; Supplementary Table 1; Supplementary Figure 6, 7).

In proteins, amino acid pairs that are remote on the primary sequence are often spatially close and interact in the $3 \mathrm{D}$ structure, ensuring the appropriate protein stability and function ${ }^{31}$. We therefore assessed whether ProteinGAN was able to learn such local and global amino acid relationships by looking for pairwise amino acid relationships across the full length of the MDH sequences. To investigate local pairwise relationships we calculated the amino acid association measures for natural and generated sequences using the minimal proximity function $Z_{m}$ (Santoni et al. 2016). The function $Z_{m}(A, B)$ counts, for each pairwise combination of the 20 amino acids, the average distance between amino acid $A$ to the next amino acid B occuring in the sequence. The calculated distances can be expressed as a matrix of all pairwise combinations (Figure 1e). The matrices for the natural and generated sequences were $88 \%$ similar, showing that the amino acid positional order had been learned by ProteinGAN, capturing the local amino acid relationships existing in natural sequences. One of the main differences between the two sequence sets was in tryptophan (Figure 1e, W column), likely resulting from the fact that $22 \%$ of the natural $\mathrm{MDH}$ sequences used did not have tryptophan. To investigate the global amino acid relationships, we calculated the pairwise amino acid frequency distributions for all combinations of position pairs in all sequences in multiple sequence alignments. These frequency distributions were then used to calculate correlations between the training and generated sequences. Overall, we found strong correlations between the natural and generated sequences (averaged Pearson's $r=0.95$, Supplementary Figure 8), 
which demonstrated that the pairwise relationships were highly similar in both sets of sequences. To expand on this, we inspected whether the generated MDH sequences possessed the two main Pfam (Finn et al. 2014) domains "Ldh_1_N" and "Ldh_1_C" that were identified (E-value < 1e-10) in the natural $\mathrm{MDH}$ sequences. Indeed, we found that $98 \%$ of the generated sequences contained both signatures, with the rest containing one of the two domains. Collectively, our results show that ProteinGAN-generated sequences are of high quality and closely mimic natural $\mathrm{MDH}$ proteins, both in terms of amino acid distributions at individual sites, as well as in terms of local and long-distance relationships between pairs of amino acids present throughout the primary sequence of the MDH family.

We then explored whether ProteinGAN was able to generalize the entire protein family beyond the training dataset, i.e. to generate novel sequence diversity. Visualization of the sequence diversity of generated and natural sequences using t-distributed stochastic neighbour embedding (t-SNE) dimensionality reduction ${ }^{32}$ showed that majority of natural MDH sequences grouped into large clusters (Figure 2a), as they were highly similar (median pairwise identity 92\%, Supplementary Figure 9). In contrast, the generated sequences grouped into smaller clusters interpolating between the natural sequence clusters and resembled a learned manifold of the MDH sequence space (Figure 2a). To assess whether the diversity in generated sequences would contain novel and functionally relevant biological properties, we performed a search of $\mathrm{CATH}^{33}$ sequence models corresponding to all known 3D structural protein domains (Methods). We first evaluated whether the network generated new structural domain diversity over the training period (Figure $2 b$ ). While the number of identified structural domains plateaued at the early stage of training (after approx. 0.2M steps), corresponding to $79 \%$ of all identified domains, additional structural CATH domains were discovered throughout the entire training process. In total, 119 novel structural sequence motifs were identified $(E$-value $<1 \mathrm{e}-6)$ in the generated sequences that do not exist in the training MDH dataset (Figure $2 \mathrm{~b}$ inset), demonstrating the network's ability to generate novel biologically relevant sequences. We next evaluated whether the generated structural domain diversity was due to chance. To test this, as a control, we randomly introduced amino acid substitutions into the natural $\mathrm{MDH}$ sequences, whilst preserving natural amino acid frequency distribution and the rate of mutations to mimic the natural sequence variability (Figure $2 \mathrm{~b}$ inset, Methods). The total structural domain diversity was reduced by $38.9 \%$ in mutated natural sequences, of which $97.4 \%$ were present in natural sequences, demonstrating that random mutations did not produce biologically relevant sequence diversity (Figure $2 \mathrm{~b}$ inset, Fisher's exact test $p$-value $<8.2 \mathrm{e}-16$ ). Using clustering analysis based on sequence similarity we observed that on average over $95 \%$ of the generated sequences were not more than $10 \%$ similar to each other ( $90 \%$ sequence identity within the cluster, Figure $2 \mathrm{c}$ ), in contrast to only $17 \%$ of the natural sequences at the same sequence identity level. This shows that the generated sequences expanded the currently known sequence space of the MDH family up to 4-fold (Figure 2c inset). 


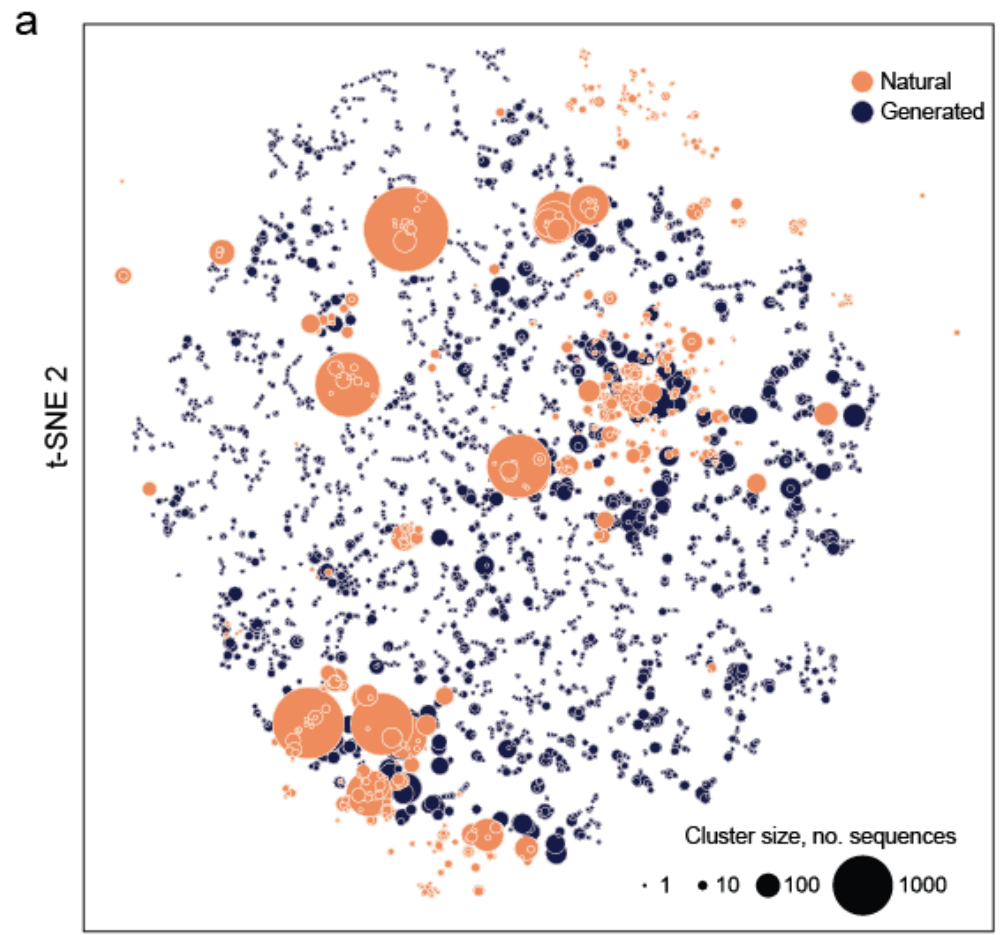

t-SNE 1
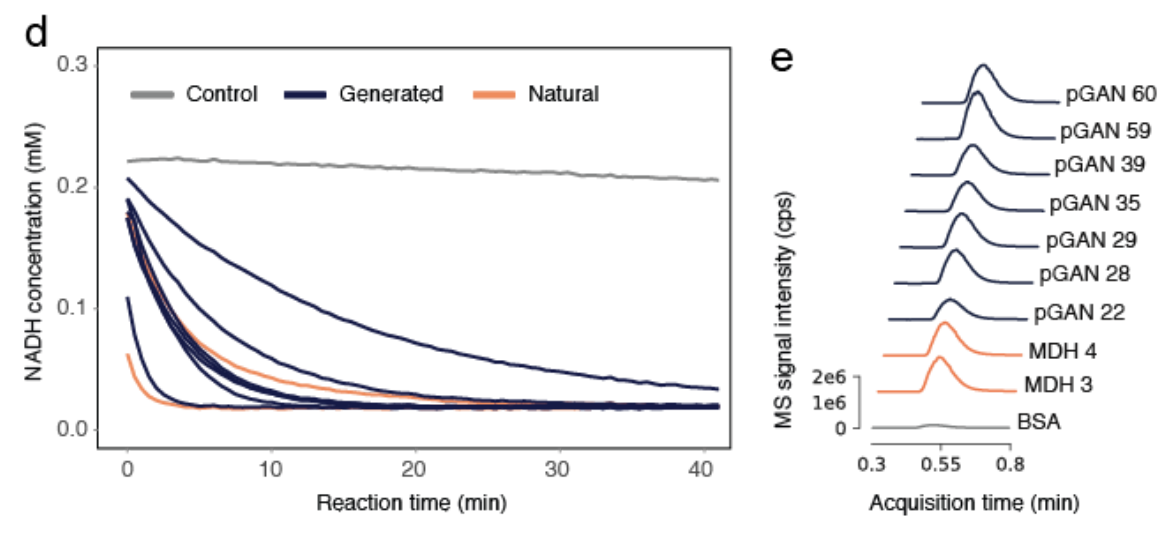

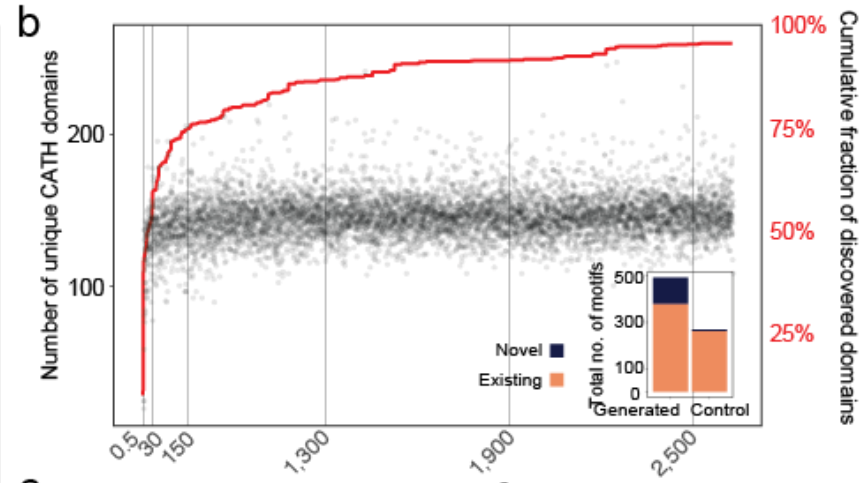

C

Training steps, $\times 10^{3}$
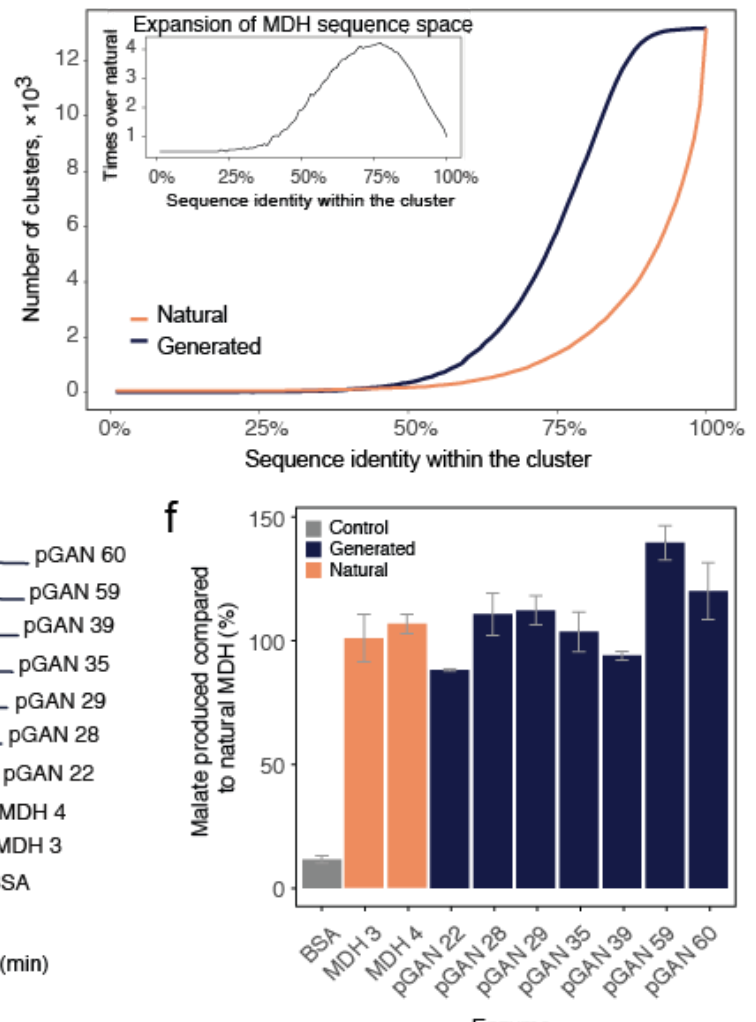

Enzyme

Figure 2. | ProteinGAN expands the functional MDH sequence space. a) The protein sequence space was visualized by transforming a distance matrix derived from $\mathrm{k}$-tuple measures of protein sequence alignment (Wilbur WJ, Lipman DJ. 1983) into a t-SNE embedding. Dot sizes represent the $70 \%$ identity cluster size for each representative. As opposed to natural sequences, generated sequences formed disparate small clusters indicating their diverse nature. b) CATH domain diversity generated throughout evolution of ProteinGAN. At every 1200 learning steps, 64 sequences were sampled and searched for representative CATH domains (E-value <1e-6). Inset: ProteinGAN generated novel domains that are not present in the existing MDH family (left). In contrast to ProteinGAN-generated sequences, randomly introducing mutations does not expand MDH sequence diversity (Fisher's exact test $p$-value $<8.2 \mathrm{e}-16$ ), but rather decreases CATH domain diversity (right). For random controls, 10 000 mutated natural sequences were simultaneously searched for presence of CATH domains (see Methods). c) Comparison of sequence diversity between generated sequences and the natural MDH dataset. By varying sequence identity cutoffs, generated sequences group into up to 4 times more clusters than natural sequences demonstrating expanded sequence diversity. Inset shows the ratio of number of clusters (Y-axis) at different sequence identity cutoffs (X-axis). d) MDH activity measured by fluorescently monitoring NADH consumption using protein expression 
method 1 (Methods). e) Catalytic activity confirmed using LC-MS/MS operating in selected reaction monitoring mode for enzymes expressed using protein expression method 1 (Methods). f) Oxaloacetate to malate conversion yields are comparable to natural MDH enzymes as determined using mass spectrometry.

Finally, considering that random amino acid substitutions typically result in a decline or even complete loss of protein activity ${ }^{4,10-16}$, we experimentally tested whether the ProteinGAN-generated MDH sequences were catalytically active in vitro. We selected 60 representative generated sequences within a range of $45 \%$ to $98 \%$ pairwise sequence identity to natural $\mathrm{MDH}$ and with 7 to 157 amino acid substitutions compared to their closest MDH neighbour (Supplementary Figure 10, Supplementary Table 2 ), of which 55 were successfully synthesized and cloned into an expression vector. Production of recombinant proteins in Escherichia coli and purification using affinity chromatography yielded 11 protein variants (Method 1) that could be purified from the cell lysate soluble fraction (Supplementary Table 3, Supplementary Figure 11). With the aim to identify additional soluble proteins, we repeated the experiment (Method 2) under growth conditions favouring protein folding and solubility using ArcticExpress E.coli strain, expanding the number of purified soluble proteins to a total of 19 (Supplementary Table 3, 35\% of all synthesized protein variants). This is comparable to other systematic studies, which typically obtain soluble protein for $20 \%$ to $40 \%$ of all tested constructs ${ }^{34-36}$. The purified proteins were assessed for MDH activity (Supplementary Figure 2) by monitoring NADH consumption using a spectrophotometer. 13 of the 19 soluble enzymes, including a variant with 106 amino acid substitutions (66\% identity to the closest existing enzyme, Supplementary Figure 12), showed MDH catalytic activity (Figure 2d, Supplementary Table 3, Supplementary Figures 13, 14). Furthermore, for the subset of 8 purified enzymes for which the protein amount could be accurately quantified the generated $\mathrm{MDH}$ proteins displayed similar reaction rates as wild-type enzymes (Supplementary Figure 13). These enzymes were also confirmed, by LC-MS/MS, to convert oxaloacetate to malate with reaction yields comparable to commercial MDH enzyme controls (Figure 2e, f).

In conclusion, here we present a generative adversarial network, ProteinGAN, that successfully captures the natural properties of proteins and enables discovery of novel functional sequences. In vitro experiments confirm that a large portion (24\%) of the generated enzymes are soluble and many display catalytic activities comparable to - or surpassing that of - natural enzymes (Figure 2d,e,f Supplementary Figure 11,13). The generated functional enzymes contain up to 106 mutations compared to the closest natural malate dehydrogenase (Supplementary Figure 12), while retaining functionally relevant sequence motifs and the correct position-specific amino acid composition that preserves long-range amino acid interactions (Figure 1). Since ProteinGAN enables large leaps to unexplored sections of the functional sequence space (Figure 2a), it opens up the biochemical exploration of the highly diverse enzymes populating this space. Such enzymes may have catalytic properties that differ significantly from those found in natural enzymes, as they have not evolved with the constraint to carry out specific functions in 
living organisms as natural enzymes have. Navigating the fitness landscape to find even one such enzyme using current methods, including random mutagenesis ${ }^{4}$ and recombination of homologous proteins ${ }^{8,9}$, would be highly laborious or not even feasible. Due to the exponential decline in protein fitness with the number of random mutations ${ }^{11,37}$ or the number of parent molecules that are used to generate the recombination libraries ${ }^{38,39}$, current methods are fundamentally limited in the sequence space that can be explored by their use. The expanded functional sequence diversity provided by ProteinGAN (Figure 2b,c) may also provide suitable, non-natural, starting points for protein engineering ${ }^{40}$, with great potential for applications in biocatalysis ${ }^{41}$. We speculate that further development of the ProteinGAN framework will enable even greater leaps in sequence space and may also make the method applicable to smaller enzyme families. Future work should explore whether ProteinGAN can be trained on entire protein families where, despite sharing sequence similarity, its members perform distinct functions. 


\section{Methods and materials}

\section{Neural network architecture details}

The GAN architecture consisted of two networks - a discriminator and a generator - each of which used ResNet blocks ${ }^{42}$ (Supplementary Figure 1). Each block in the discriminator contained 3 convolution layers with filter size of $3 \times 3,2$ batch normalization layers ${ }^{43}$ and leaky ReLU activations ${ }^{44}$. The generator residual blocks consisted of two transposed convolution layers, one convolution layer with the same filter size of $3 \times 3$ and leaky ReLU activations. Each network had one self-attention layer ${ }^{30}$. Transposed convolution technique was chosen for up-sampling, as it yielded the best results experimentally (Supplementary Figure 15). For loss, non-saturating loss with $\mathrm{R} 1$ regularization ${ }^{45}$ was used (Supplementary Figure 16). To ensure training stability, Spectral normalization ${ }^{46}$ was implemented in all layers.

The input to the discriminator was one-hot encoded with a vocabulary size of 21 (20 canonical amino acids and a sign that denoted a space at the beginning or end of the sequence). The generator input was a vector of 128 values that were drawn from a random distribution with mean 0 and standard deviation of 0.5 , with the exception that values whose magnitude was more than 2 standard deviations away from the mean were re-sampled. The dimensions of generated outputs were $512 \times 21$, wherein some of the positions denoted spaces. The implementation of ProteinGAN can be accessed at https://github.com/biomatterdesigns/ProteinGAN.

\section{Network training data}

Bacterial malate dehydrogenase (MDH) sequences were downloaded from Uniprot on January 10th 2019 47. Sequences longer than 512 amino acids or containing non-canonical amino acids were filtered out. The final dataset consisted of 16898 sequences, which were clustered into $70 \%$ identity clusters using the MMseq2 tool ${ }^{48}$ for balancing the dataset during the training process. $20 \%$ of the clusters with less than 3 sequences were randomly selected for validation (192 sequences) and the rest of the dataset was used for training (16 706 sequences).

\section{Network training process}

The ratio $1: 1$ between generator and discriminator training steps was selected (Supplementary materials, Supplementary Figure 17). ADAM algorithm ${ }^{49}$ was used to optimize both networks. Throughout the training, the learning rate was gradually decreased from $1 \mathrm{e}-3$ to $5 \mathrm{e}-5$ for both the generator and the discriminator. To avoid bias towards sequences with large number of homologues, smaller clusters were dynamically up-sampled during the training. In order to track the performance, along with GAN losses, 
generated data was constantly evaluated. Without halting the training process, every 1200 steps generated sequences were automatically aligned with the training and validation datasets using BLAST 50,51. Throughout the training, BLOSUM45, E-value and identity scores as well as standard deviation of the discriminator layer were calculated and monitored (Supplementary Figure 3, 18, 19, 20). ProteinGAN was trained for $2.5 \mathrm{M}$ steps with a batch size of 64 (one step consisted of 64 sequences). The training took 210 hours ( 9 days) on NVIDIA Tesla P100 (16 GB).

\section{Generated sequences bioinformatic analysis}

Multiple sequence alignments Multiple sequence alignments (MSA) (Figure 1) were calculated using using Clustal Omega ${ }^{52}$ by merging natural and generated datasets in equal amounts. To calculate further Shanon entropies, after MSA we split the alignment corresponding to generated and training sequences. Columns having more than $75 \%$ of gaps in either dataset were removed from further analysis. For each column in MSA, Shannon entropy was calculated as follows: s.e. $=-\sum_{i=1}^{20} p\left(x_{i}\right) \log _{20} p\left(x_{i}\right)$, where $p\left(x_{i}\right)$ is the frequency of amino acid $i$ occurring at a column of MSA.

Amino acid pair association matrices Amino acid pair association matrices were calculated for every possible pair in a sequence and averaged over the whole dataset. The association score was used as reported in the original article (Santoni et al. 2016), where $Z_{m}$ is expressed as follows:

$Z_{m}(a, b)=\frac{P_{m}(a, b)-\overline{P_{m}(a, \operatorname{Rand}(b))}}{\sigma_{P_{m}(a, \operatorname{Rand}(b))}}$. Here $\overline{P_{m}(a, \operatorname{Rand}(b))}$ and $\sigma_{P_{m}(a, \operatorname{Rand}(b))}$ are the average and the standard deviation of the randomly shuffled sequence association score for the same pair. The association function for scoring was selected as the minimal proximity function:

$P_{m}(a, b)=\frac{1}{n} \sum_{i=1}^{n} \min _{j=1, \ldots, m}\left\{\left|x_{i}-y_{i}\right|\right\}$. Here for each position $x_{i}$ of amino acid $a$, the closest occurrence of amino acid $b$ at position $y_{i}$ is identified and the average of the distances between the pairs is calculated. In our implementation, if a sequence does not contain a certain amino acid, Null value is returned for the pairs containing the amino acid.

Sequence clustering Sequence clustering was performed using MMseqs2 ${ }^{48}$ with easy-cluster option and required sequence identity cutoff.

Pfam/CATH domain search All sequences generated by ProteinGAN were classified using HMMER3 ${ }^{53}$ search over Pfam 32.0 database ${ }^{54}$. HMMs for each CATH representative domain from the sequence clusters at $35 \%$ sequence identity (v4.1) were downloaded from CATH database repository ${ }^{33}$. To avoid biases in sequence scoring, generated sequences together with the natural sequences in equal quantities were appended to the same file and in all tests were searched simultaneously using hmmsearch tool with 
default options ${ }^{53}$. To evaluate whether generated domain diversity was not due to chance, we chose a random subset of 10000 natural sequences and mutated them by randomly introducing on average $100 \pm$ 30 sd substitutions of amino acids (corresponding to median identity of generated sequences Figure $1 \mathrm{~b}$ ) that were uniformly sampled of natural amino acid probability distribution. Generated, natural and mutated sequences (10 000 of each) were searched as one database and hits were considered significant with E-value $<1 \mathrm{e}-6$. The analyzed sequences were generated by latest checkpoint model $(\sim 2.5 \mathrm{M}$ training steps).

t-SNE plot generation A distance matrix of cluster representatives was used as the t-SNE input. To get cluster representatives, first, the number of sequences in both datasets were equalized by taking 13,272 sequences from natural and generated datasets. These sequences were independently clustered using MMseqs $2{ }^{48}$ with $70 \%$ minimal sequence identity. This generated 926 clusters of natural sequences and 3,778 clusters of generated sequences. Representative sequences of these clusters were chosen based on MMseqs2 output. From the representative sequences a distance matrix was generated using Clustal Omega ${ }^{52,55}$. The distance matrix was used with the scikit-learn t-SNE module ${ }^{56}$ with default settings (early exaggeration 12, learning rate 200, maximum number of iterations: 1000) except that the embedding generation perplexity was set to 7 . Coordinates given by t-SNE were used for plotting, the size of a given dot was visualized based on the cluster size it represents.

Visualization of GAN training Sequences generated during the training period were sampled at 14 different times. For each of the 14 checkpoints, 64 sequences were taken, every checkpoint was taken after $i(x)=2^{x} * 300$ (where $\mathrm{x}$ is number of checkpoints) GAN steps, with the first checkpoint replaced with 1 instead of 300 . For all the generated sequences, a global identity to the closest sequence in training dataset was calculated. Identities of each checkpoint were plotted.

Correlation of distant dimer pairs To calculate the correlation of close and distant dimer pairs between the datasets, the total number of individual dimers for every possible MSA position pair was calculated: $d s=\left\{m_{a 1, a 1}, m_{a 1, a 2}, \ldots, m_{a 21, a 21}\right\}$, here $m_{a i, a j}$ was a set of dimer $a_{i} a_{j}$ counts over each position pair of a multiple sequence alignment $\left(m_{a i, a j}=\left\{d_{1,1}, d_{1,2}, \ldots, d_{n, n}\right\}\right)$. Each number of $m_{a i, a j}$ set was calculated by summing $a_{i} a_{j}$ dimers over all sequences of MSA in positions $n$ and $m: d_{n, m}=\sum_{z=1}^{s} a_{i} a_{j}$ here $s$ is the total number of sequences in MSA. ds was calculated for each dataset and for each member of the ds set, Pearson's $r$ was calculated between the datasets (natural and generated). These correlations were plotted as a heatmap (Supplementary Figure 8). For $d s$ calculation only columns containing less than $75 \%$ of gaps in both natural or generated datasets were used.

Experimental validation of generated enzymes The sequences generated by ProteinGAN were 
synthesized, cloned into the pET21a expression vector and sequence-verified by Twist Bioscience. In addition to the enzyme sequence a C-terminal linker and four histidines (AAALEHHHH) were added, resulting in a deca-His-tag in the final construct (which includes six histidines derived from the expression vector), to enable downstream affinity purification. Method 1: The constructs were transformed into the BL21(DE3) E. coli expression strain. From the resulting transformation mixture $15 \mu$ was used to inoculate $500 \mu \mathrm{LB}$ broth supplemented with $100 \mu \mathrm{g} / \mathrm{ml}$ carbenicillin. Cells were grown overnight at $32^{\circ} \mathrm{C}$ in a 96 deep well plate with $700 \mathrm{rpm}$ orbital shaking. Protein expression was achieved by diluting the overnight cultures 1:30 into $1 \mathrm{ml}$ autoinduction TB including trace elements (Formedium, UK) supplemented with $100 \mu \mathrm{g} / \mathrm{ml}$ carbenicillin and grown for $4 \mathrm{~h}$ at $37^{\circ} \mathrm{C}$, followed by overnight growth at $18^{\circ} \mathrm{C}$ and $700 \mathrm{rpm}$ shaking. Cells were collected by centrifugation and the cell pellets frozen at $-80^{\circ} \mathrm{C}$ overnight. To purify the recombinant proteins, cells were thawed, resuspended in $200 \mu$ lysis buffer (50 mM HEPES pH 7.4, 5\% glycerol, $300 \mathrm{mM} \mathrm{NaCl}, 0.5 \mathrm{mM}$ TCEP, $0.5 \mathrm{mg} / \mathrm{ml} \mathrm{lysozyme,} 10 \mathrm{U} / \mathrm{ml}$ DNasel, 2 $\mathrm{mM} \mathrm{MgCl} 2$ ), and incubated for $30 \mathrm{~min}$ at room temperature. To improve lysis triton-X-100 was added to a final concentration of $0.125 \%(\mathrm{v} / \mathrm{v})$, and the cells were frozen at $-80^{\circ} \mathrm{C}$ for $30 \mathrm{~min}$. After thawing in a room temperature water bath, the lysates were spun down for $10 \mathrm{~min}$ at $3000 \mathrm{xg}$ to remove cell debris, and the supernatants were transferred to a new 96-well plate with $50 \mu \mathrm{l}$ Talon resin in each well (Takara Bio, Japan). Unspecific binding of proteins to the resin was reduced by adding imidazole to a final concentration of $10 \mathrm{mM}$ in each well. The plate was incubated at room temperature for 30 min with 400 rpm shaking, after which the lysates with the beads were transferred to a 96-well filter plate (Thermo Scientific, USA, Nunc 96-well filter plates), placed over a 96-well collection plate, and centrifuged for 1 $\min$ at $500 \mathrm{xg}$ in a swing-out centrifuge. The resin was washed three times with $200 \mu \mathrm{l}$ wash buffer (50 $\mathrm{mM}$ HEPES pH 7.4, 5\% glycerol, $300 \mathrm{mM} \mathrm{NaCl}, 0.5 \mathrm{mM}$ TCEP, $40 \mathrm{mM}$ imidazole), and the proteins were

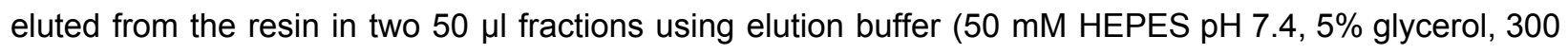
$\mathrm{mM} \mathrm{NaCl}, 0.5 \mathrm{mM}$ TCEP, $250 \mathrm{mM}$ imidazole). The two eluate fractions were combined and transferred to a 96-well desalting plate (Thermo Scientific, USA, Zeba Spin Desalting Plate, 7K MWCO) pre-equilibrated with sample buffer (50 mM HEPES pH 7.4, $\%$ glycerol, $300 \mathrm{mM} \mathrm{NaCl}, 0.5 \mathrm{mM}$ TCEP). The plate was spun down $1000 \times \mathrm{g}$ for $1 \mathrm{~min}$, and collected proteins were analysed by SDS-PAGE followed by Coomassie staining. The soluble proteins were carried on for further characterisation.

To test for malate dehydrogenase activity, an aliquot of purified protein was added to a reaction mixture containing $0.15 \mathrm{mM} \mathrm{NADH}, 0.2 \mathrm{mM}$ oxaloacetic acid and $20 \mathrm{mM}$ HEPES buffer ( $\mathrm{pH}$ 7.4). The final reaction volume was $100 \mu \mathrm{l}$ and the reaction was carried out at room temperature in a UV-transparent 96-well half-area plate (UV-Star Microplate, Greiner, Austria). Activity was measured in triplicates by following $\mathrm{NADH}$ oxidation to NAD+, with absorbance reading at $340 \mathrm{~nm}$ performed every $30 \mathrm{sec}$ for 15 min in a BMG Labtech SPECTROstar Nano spectrophotometer. Un-specific oxidation of NADH was monitored in no-substrate controls, and these values were subtracted from the other samples. Conversion 
from absorption values to NADH concentration was carried out using an extinction coefficient of $6.22 \mathrm{mM}$. For calculation of kinetic parameters, $10 \mathrm{nM}$ of each protein was assayed with a range of oxaloacetate concentrations.

LC-MS/MS quantification was performed for selected active enzymes. The activity assay was performed as outlined above, in triplicates, with protein concentrations ranging between 10 and $250 \mathrm{nM}$. Reactions were terminated after $45 \mathrm{~min}$ by diluting the assay mixtures in water to $1 \mu \mathrm{g} / \mathrm{ml}$ starting concentration of oxaloacetate. For chromatographic separation a Zorbax Eclipse Plus C18 $50 \mathrm{~mm} \times 2.1 \mathrm{~mm} \times 1.8 \mu \mathrm{m}$ (Agilent) with an Nexera series HPLC (Shimadzu) was used. Mobile phase A was composed of H2O (MiliQ HPLC grade) with $0.1 \%$ Formic acid (Sigma); mobile phase B was Methanol (Sigma) with $0.1 \%$ Formic acid (Sigma). The oven temperature was $40^{\circ} \mathrm{C}$. The chromatographic gradient was set to consecutively increase from $0 \%$ to $100 \%$, hold, decrease from $100 \%$ to $0 \%$ and hold, in $60 \mathrm{sec}, 30 \mathrm{sec}$, $30 \mathrm{sec}$ and $30 \mathrm{sec}$, respectively. The autosampler temperature was $15^{\circ} \mathrm{C}$ and the injection volume was $0.5 \mu \mathrm{L}$ with full loop injection. For MS quantification a QTRAP® 6500 System (Sciex) was used, operating in negative mode with Multiple Reaction Monitoring (MRM) parameters optimized for Malic acid based on published parameters ${ }^{57}$. Electrospray ionization parameters were optimized for $0.8 \mathrm{~mL} / \mathrm{min}$ flow rate, and were as follows: electrospray voltage of $-4500 \mathrm{~V}$, temperature of $500{ }^{\circ} \mathrm{C}$, curtain gas of 40 , CAD gas set to Medium, and gas 1 and 2 of 50 and 50 psi, respectively. The instrument was mass calibrated with a mixture of polypropylene glycol (PPG) standards. The software Analyst 1.7 (Sciex) and MultiQuant 3 (Sciex) was used for analysis and quantitation of results, respectively.

Additionally (Method 2), to obtain additional soluble proteins, MDH constructs were transformed into ArcticExpress competent cells (Agilent technologies, USA). The transformants were inoculated into 500 $\mu \mathrm{L}$ of LB media with $15 \mu \mathrm{g} / \mathrm{mL}$ of Gentamicin and $50 \mu \mathrm{g} / \mathrm{mL}$ of Ampicillin and grown overnight at $30^{\circ} \mathrm{C}$ in a Thermomixer Comfort Eppendorf thermomixer (Eppendorf, Germany). $250 \mu \mathrm{L}$ of overnight culture were transferred to $10 \mathrm{~mL}$ (dilution 1:40) of semi-synthetic media (1 \% Tryptone, 0.5\% Yeast extract, $0.268 \%$ $\left(\mathrm{NH}_{4}\right)_{2} \mathrm{SO}_{4}, 0.15 \% \mathrm{NH}_{4} \mathrm{Cl}, 0.6 \% \mathrm{KH}_{2} \mathrm{PO}_{4}, 0.4 \% \mathrm{~K}_{2} \mathrm{HPO}_{4}, 1 \%$ Glycerol, $\mathrm{pH} 7.0$ ) supplemented with 15 $\mu \mathrm{g} / \mathrm{mL}$ of Gentamicin and $50 \mu \mathrm{g} / \mathrm{mL}$ of Ampicillin. Cells were cultivated at $37^{\circ} \mathrm{C}$ for 2 hours, until $\mathrm{OD}_{600}$ reached $0.6-0.8$, then the media were enriched with $0.5 \mathrm{M}$ of saccharose. Induction was carried out at $12^{\circ} \mathrm{C}$ with $0.5 \mathrm{mM} \mathrm{IPTG}$ overnight. The cells were harvested by centrifugation ( $4000 \mathrm{x} \mathrm{g} 10 \mathrm{~min}$ at $4^{\circ} \mathrm{C}$ ), resuspended in $0.1 \mathrm{M}$ potassium phosphate buffer, $\mathrm{pH} 7.0$ and then sonicated on ice in $2.0 \mathrm{~mL}$ tubes at $30 \%$ amplitude for $5 \mathrm{~min}$ of total ON time (30 s on/30 s off) by using the Bandelin SonoPuls HD 2070 homogeniser (BANDELIN, Germany).

To remove cell debris, the lysates were centrifuged at $16000 \times \mathrm{g}, 4^{\circ} \mathrm{C}$. The soluble recombinant $\mathrm{MDH}$ mutants were purified by using HisPur ${ }^{\mathrm{TM}}$ Ni-NTA Spin columns (ThermoFisher Scientific, USA). The 
columns with loaded supernatants were washed with Wash buffer (0.1 M Potassium phosphate buffer, $\mathrm{pH} 7.4, \mathrm{NaCl} 250 \mathrm{mM}$ and $40 \mathrm{mM}$ imidazole). Proteins were eluted with Elution buffer (0.1 M Potassium phosphate buffer, $\mathrm{pH} 7.4, \mathrm{NaCl} 250 \mathrm{mM}$ and $300 \mathrm{mM}$ ). The eluted fractions were dialysed against $0.1 \mathrm{M}$ Potassium phosphate buffer, $\mathrm{pH}$ 7.4. The concentration of the proteins was determined using NanoDrop 2000 (Thermofisher Scientific, USA). The aliquots of total lysate, soluble lysate fraction and purified protein were loaded onto SDS PAGE $15 \%$.

The malate dehydrogenase activity was measured at $25^{\circ} \mathrm{C}$ in 96 -well flat bottom UV transparent plate (UV-Star Microplate) (Greiner Bio-One, Austria). The reaction mixture (final volume $200 \mu \mathrm{L}$ ) contained an aliquot of purified protein, freshly prepared $0.15 \mathrm{mM} \mathrm{NADH}$ and $0.2 \mathrm{mM}$ oxaloacetic acid, and $0.1 \mathrm{M}$ Potassium phosphate buffer, $\mathrm{pH}$ 7.4. The absorbance reading was done at $340 \mathrm{~nm}$ every 5 seconds for 3 minutes in a BioTek PowerWave XS microplate reader (Biotek, USA). For NADH at $340 \mathrm{~nm} 6.22 \mathrm{mM} \mathrm{cm}^{-1}$ extinction coefficient $(\varepsilon M)$ was used. The path length $(I)$ in the microplate was calculated according $A=c x$ $\varepsilon M \times I$.

\section{Conflicts of interest}

Laurynas Karpus, Vykintas Jauniškis, Donatas Repecka and Rolandas Meskys are co-founders and shareholders of the company Biomatter Designs. The other authors declare no commercial or financial conflict of interest.

\section{Acknowledgements}

We thank Greta Stonyte, Juozas Nainys, Simran Aulakh and Clara Correia-Melo for comments on the manuscript. L.K. and R.M. were supported by the Agency for Science, Innovation and Technology (Lithuania) grants No. 31V-59/(1.78)SU-1687 and 01.2.2-MITA-K-702-04-0001. A.Z. is a SciLifeLab fellow. 


\section{References}

1. Pierce, N. A. \& Winfree, E. Protein design is NP-hard. Protein Eng. 15, 779-782 (2002).

2. Mandecki, W. The game of chess and searches in protein sequence space. Trends in Biotechnology 16, 200-202 (1998).

3. Goodfellow, I. et al. Generative Adversarial Nets. in Advances in Neural Information Processing Systems 27 (eds. Ghahramani, Z., Welling, M., Cortes, C., Lawrence, N. D. \& Weinberger, K. Q.) 2672-2680 (Curran Associates, Inc., 2014).

4. Romero, P. A. \& Arnold, F. H. Exploring protein fitness landscapes by directed evolution. Nat. Rev. Mol. Cell Biol. 10, 866-876 (2009).

5. Keefe, A. D. \& Szostak, J. W. Functional proteins from a random-sequence library. Nature 410, 715-718 (2001).

6. Taverna, D. M. \& Goldstein, R. A. Why are proteins marginally stable? Proteins 46, 105-109 (2002).

7. Axe, D. D. Estimating the prevalence of protein sequences adopting functional enzyme folds. J. Mol. Biol. 341, 1295-1315 (2004).

8. Hansson, L. O., Bolton-Grob, R., Massoud, T. \& Mannervik, B. Evolution of differential substrate specificities in Mu class glutathione transferases probed by DNA shuffling 1 1Edited by R. Huber. Journal of Molecular Biology 287, 265-276 (1999).

9. Crameri, A., Raillard, S. A., Bermudez, E. \& Stemmer, W. P. DNA shuffling of a family of genes from diverse species accelerates directed evolution. Nature 391, 288-291 (1998).

10. Bloom, J. D., Labthavikul, S. T., Otey, C. R. \& Arnold, F. H. Protein stability promotes evolvability. Proc. Natl. Acad. Sci. U. S. A. 103, 5869-5874 (2006).

11. Guo, H. H., Choe, J. \& Loeb, L. A. Protein tolerance to random amino acid change. Proceedings of the National Academy of Sciences 101, 9205-9210 (2004).

12. Rennell, D., Bouvier, S. E., Hardy, L. W. \& Poteete, A. R. Systematic mutation of bacteriophage T4 lysozyme. J. Mol. Biol. 222, 67-88 (1991).

13. Axe, D. D., Foster, N. W. \& Fersht, A. R. A Search for Single Substitutions That Eliminate Enzymatic Function in a Bacterial Ribonuclease†. Biochemistry 37, 7157-7166 (1998).

14. Shafikhani, S., Siegel, R. A., Ferrari, E. \& Schellenberger, V. Generation of large libraries of random mutants in Bacillus subtilis by PCR-based plasmid multimerization. Biotechniques 23, 304-310 (1997).

15. Rockah-Shmuel, L., Tóth-Petróczy, Á. \& Tawfik, D. S. Systematic Mapping of Protein Mutational Space by Prolonged Drift Reveals the Deleterious Effects of Seemingly Neutral Mutations. PLoS Comput. Biol. 11, e1004421 (2015).

16. Sarkisyan, K. S. et al. Local fitness landscape of the green fluorescent protein. Nature 533, 397-401 (2016).

17. Yang, K. K., Wu, Z. \& Arnold, F. H. Machine-learning-guided directed evolution for protein engineering. Nat. Methods 16, 687-694 (2019).

18. AIQuraishi, M. End-to-End Differentiable Learning of Protein Structure. Cell Syst 8, 292-301.e3 (2019).

19. Rives, A. et al. Biological structure and function emerge from scaling unsupervised learning to 250 million protein sequences. doi:10.1101/622803

20. Riesselman, A. J., Ingraham, J. B. \& Marks, D. S. Deep generative models of genetic variation capture the effects of mutations. Nat. Methods 15, 816-822 (2018).

21. Alley, E. C., Khimulya, G., Biswas, S., AlQuraishi, M. \& Church, G. M. Unified rational protein engineering with sequence-only deep representation learning. doi:10.1101/589333

22. Romero, P. A., Krause, A. \& Arnold, F. H. Navigating the protein fitness landscape with Gaussian processes. Proc. Natl. Acad. Sci. U. S. A. 110, E193-201 (2013).

23. Bileschi, M. L. et al. Using Deep Learning to Annotate the Protein Universe. bioRxiv 626507 (2019). doi:10.1101/626507

24. Karras, T., Laine, S. \& Aila, T. A Style-Based Generator Architecture for Generative Adversarial Networks. (2018). 
25. Alonso, E., Moysset, B. \& Messina, R. Adversarial Generation of Handwritten Text Images Conditioned on Sequences. (2019).

26. van den Oord, A. et al. WaveNet: A Generative Model for Raw Audio. (2016).

27. Gupta, A. \& Zou, J. Feedback GAN (FBGAN) for DNA: a Novel Feedback-Loop Architecture for Optimizing Protein Functions. (2018).

28. Killoran, N., Lee, L. J., Delong, A., Duvenaud, D. \& Frey, B. J. Generating and designing DNA with deep generative models. arXiv [cs.LG] (2017).

29. Bai, S., Kolter, J. Z. \& Koltun, V. An Empirical Evaluation of Generic Convolutional and Recurrent Networks for Sequence Modeling. (2018).

30. Zhang, H., Goodfellow, I., Metaxas, D. \& Odena, A. Self-Attention Generative Adversarial Networks. (2018).

31. Marks, D. S. et al. Protein 3D structure computed from evolutionary sequence variation. PLoS One 6, e28766 (2011).

32. Maaten, L. van der \& Hinton, G. Visualizing Data using t-SNE. J. Mach. Learn. Res. 9, 2579-2605 (2008).

33. Dawson, N. L. et al. CATH: an expanded resource to predict protein function through structure and sequence. Nucleic Acids Res. 45, D289-D295 (2017).

34. Huang, $\mathrm{H}$. et al. Panoramic view of a superfamily of phosphatases through substrate profiling. Proc. Natl. Acad. Sci. U. S. A. 112, E1974-83 (2015).

35. Pertusi, D. A., Stine, A. E., Broadbelt, L. J. \& Tyo, K. E. J. Efficient searching and annotation of metabolic networks using chemical similarity. Bioinformatics 31, 1016-1024 (2015).

36. Mashiyama, S. T. et al. Large-Scale Determination of Sequence, Structure, and Function Relationships in Cytosolic Glutathione Transferases across the Biosphere. (2014). doi:10.1371/journal.pbio.1001843

37. Bloom, J. D. et al. Thermodynamic prediction of protein neutrality. Proc. Natl. Acad. Sci. U. S. A. 102, 606-611 (2005).

38. Neylon, C. Chemical and biochemical strategies for the randomization of protein encoding DNA sequences: library construction methods for directed evolution. Nucleic Acids Res. 32, 1448-1459 (2004).

39. Voigt, C. A., Martinez, C., Wang, Z.-G., Mayo, S. L. \& Arnold, F. H. Protein building blocks preserved by recombination. Nat. Struct. Biol. 9, 553-558 (2002).

40. Chen, T. \& Romesberg, F. E. Directed polymerase evolution. FEBS Lett. 588, 219-229 (2014).

41. Truppo, M. D. Biocatalysis in the Pharmaceutical Industry: The Need for Speed. ACS Med. Chem. Lett. 8, 476-480 (2017).

42. He, K., Zhang, X., Ren, S. \& Sun, J. Deep Residual Learning for Image Recognition. (2015).

43. loffe, S. \& Szegedy, C. Batch Normalization: Accelerating Deep Network Training by Reducing Internal Covariate Shift. (2015).

44. Maas, A. L. Rectifier Nonlinearities Improve Neural Network Acoustic Models. (2013).

45. Mescheder, L., Geiger, A. \& Nowozin, S. Which Training Methods for GANs do actually Converge? (2018).

46. Miyato, T., Kataoka, T., Koyama, M. \& Yoshida, Y. Spectral Normalization for Generative Adversarial Networks. (2018).

47. UniProt Consortium. UniProt: a worldwide hub of protein knowledge. Nucleic Acids Res. 47, D506-D515 (2019).

48. Steinegger, M. \& Söding, J. MMseqs2 enables sensitive protein sequence searching for the analysis of massive data sets. Nat. Biotechnol. 35, 1026-1028 (2017).

49. Kingma, D. P. \& Ba, J. Adam: A Method for Stochastic Optimization. (2014).

50. pubmeddev \& Altschul SF, E. al. Basic local alignment search tool. - PubMed - NCBI. Available at: https://www.ncbi.nlm.nih.gov/pubmed/2231712?dopt=Citation. (Accessed: 5th August 2019)

51. Altschul, S. F., Gish, W., Miller, W., Myers, E. W. \& Lipman, D. J. Basic local alignment search tool. J. Mol. Biol. 215, 403-410 (1990).

52. Sievers, F. et al. Fast, scalable generation of high-quality protein multiple sequence alignments using Clustal Omega. Mol. Syst. Biol. 7, 539 (2011).

53. Eddy, S. R. Accelerated Profile HMM Searches. PLoS Comput. Biol. 7, e1002195 (2011). 
bioRxiv preprint doi: https://doi org/10.1101/789719; this version posted October 4,2019 . The copyright holder for this preprint (which was not certified by peer review) is the author/funder, who has granted bioRxiv a license to display the preprint in perpetuity. It is made available under aCC-BY-NC-ND 4.0 International license.

54. El-Gebali, S. et al. The Pfam protein families database in 2019. Nucleic Acids Res. 47, D427-D432 (2019).

55. Sievers, F. \& Higgins, D. G. Clustal Omega for making accurate alignments of many protein sequences. Protein Science 27, 135-145 (2018).

56. Pedregosa, F. et al. Scikit-learn: Machine Learning in Python. J. Mach. Learn. Res. 12, 2825-2830 (2011).

57. McCloskey, D. \& Ubhi, B. K. Quantitative and Qualitative Metabolomics for the Investigation of Intracellular Metabolism. SCIEX Tech Note 1-11 (2014). 